\title{
Riesgos Hacia los Trabajadores y Comunidad por Liberaciones Accidentales de Gases Densos Peligrosos en la Ciudad de Los Angeles (Chile)
}

\author{
RISKS TO WORKERS AND THE COMMUNITY FOR ACCIDENTAL RELEASES OF HAZARDOUS DENSE GASES IN \\ LOS ANGELES CITY (CHILE).
}

\begin{abstract}
Adrián Silva', Margarita Barrandeguy², Yenny Pincheira ${ }^{3}$
1. Profesor Asistente del Departamento de Ciencias y Tecnología Vegetal. Escuela de Ciencias y Tecnologías. Universidad de Concepción. Los Ángeles. Chile.

2. Profesor Asistente del Departamento de Ciencias y Tecnología Vegetal. Escuela de Ciencias y Tecnologías. Universidad de Concepción. Los Ángeles. Chile.

3. Departamento de Ciencias y Tecnología Vegetal. Escuela de Ciencias y Tecnologías. Universidad de Concepción. Los Ángeles. Chile.
\end{abstract}

\section{RESUMEN}

Se evaluaron riesgos hacia los trabajadores y comunidad por posibles liberaciones accidentales de gases densos peligrosos, desde fuentes de uso y almacenamiento, dentro del radio urbano de Los Ángeles (Chile). Se obtuvo información desde organizaciones públicas, privadas y por trabajo de campo para determinar la ubicación de las fuentes, especificaciones y cantidades de gases. Se consideraron comercializadoras de gases industriales e instalaciones de GLP con estanques mayores o iguales a $2 \mathrm{~m}^{3}$. Se aplicaron las metodologías de evaluación de riesgos de la NTP № 937 del INSHT (España) y del Programa APELL del PNUMA.

Los gases identificados en las comercializadoras fueron $\mathrm{Ar}, \mathrm{CO}_{2}, \mathrm{~N}_{2} \mathrm{O}$ y $\mathrm{C}_{2} \mathrm{H}_{2}$. Del análisis utilizando la metodología NTP $\mathrm{N}^{\circ} 937$ se obtuvo, para los trabajadores evaluados en locales de venta de gases industriales, un nivel de riesgo a priori bajo y una prioridad de acción clase 3 y, en las instalaciones de GLP de distribución masiva, se obtuvo un riesgo moderado con prioridad de acción clase 2 . El análisis con metodología APELL arroja que las instalaciones de gas licuado, frente a una fuga de gas, tendrían para la comunidad nivel de consecuencias limitadas, graves $y$ muy graves en el 67, 29 y 3,8\% de los casos, respectivamente.

(Silva A, Barrandeguy M, Pincheira Y, 2016. Riesgos Hacia los Trabajadores y Comunidad por Liberaciones Accidentales de Gases Densos Peligrosos en la Ciudad de Los Ángeles (Chile). Cienc Trab. May-Ago; 18 [56]: 81-86).

Palabras claves: RIESGOS LABORALES, FUGA DE GASES, GASES DENSOS PELIGROSOS, GLP, APELL.

\section{ABSTRACT}

Risks to workers and the community were evaluated for possible accidental releases of hazardous dense gases from sources use and storage, within the urban area in Los Angeles (Chile). Information was obtained from public and private organizations and the fieldwork to determine the location of the sources, specifications and quantities of gases. Gases trading industries and installations of LPG tanks greater than or equal to $2 \mathrm{~m}^{3}$ were considered. Methodologies for risk assessment of the NTP No937 INSHT (Spain) and UNEP APELL program were applied.

The identified gases in the trading industries were $\mathrm{Ar}, \mathrm{CO}_{2}, \mathrm{~N}_{2} \mathrm{O}$ and $\mathrm{C}_{2} \mathrm{H}_{2}$. From the analysis which used the methodology NTP No. 937, for workers evaluated in stores selling industrial gases, a low priori risk level was obtained and a priority for action class 3. In the LPG installations of mass distribution, a moderate risk with priority action class 2 was obtained. The APELL methodology analysis reveals that the liquefied gas installations, in case of gas leaking, would have limited, serious and very serious consequences for the community in $67 \%, 29 \%$ and $3,8 \%$ of cases respectively.

Keywords: OCCUPATIONAL HAZARDS, GAS LEAKING, DANGEROUS DENSE GASES, LPG, APELL.

\section{INTRODUCCIÓN}

Muchos de los productos químicos utilizados en las operaciones industriales involucradas presentan riesgos de accidentes. ${ }^{1}$ Un factor que influye en la gravedad de éstos es el crecimiento desordenado

Correspondencia / Correspondence:

Adrián Silva F.

Juan Antonio Coloma 0201

Los Ángeles. Chile

Tel.: 56432405137

e-mail: asilvaf@udec.cl

Recibido: 10 de Abril de 2016 / Aceptado: 14 de Mayo de 2016

tanto de las grandes ciudades como de las numerosas ciudades intermedias emergentes en América Latina, lo que conlleva a un proceso de concentración del riesgo cada vez más urbano ${ }^{2}$, con la posibilidad de ocurrencia de incidentes con potencial de daño para las personas y el medio ambiente, debido al uso y/o almacenamiento de sustancias químicas. Este tipo de accidentes se viene presentando desde hace muchos años; uno de los casos más graves a nivel mundial ocurrió en la ciudad de Bhopal, India, en 1984, al producirse una fuga de isocianato de metilo desde una fábrica de pesticidas, gas tóxico que ocasionó la muerte de miles de personas. ${ }^{3}$ Asimismo, en Chile existen varios registros de daños al medio ambiente y a la salud de las personas producidos por eventos accidentales, muchos de los cuales se deben a una deficiente gestión de seguridad. ${ }^{4}$ Se pueden producir escapes tóxicos procedentes de instalaciones peligrosas fijas o del transporte y distribución de sustancias químicas ${ }^{5}$, donde el comportamiento de la nube formada dependerá de las características de las 
sustancias liberadas a la atmósfera. La densidad, por ejemplo, condiciona la evolución de un gas/vapor, de tal modo que cuando se derrama un gas "denso", el comportamiento inicial difiere sustancialmente de un gas neutro ${ }^{6,7}$, puesto que el gas denso se desplaza a nivel del suelo. Asimismo, el viento es una variable importante a considerar, debido a que los accidentes más severos han ocurrido en condiciones de vientos de baja intensidad, ya que una intensidad alta facilita la dispersión del gas. ${ }^{8}$

El transporte, almacenamiento y procesamiento de los materiales utilizados representan niveles de riesgos debido a las altas temperaturas, presiones, reactividad y/o toxicidad frecuentemente encontradas en los procesos, y que en muchos casos no son evaluados adecuadamente, ni son considerados en la toma de decisiones $^{9}$, por lo que presentan diversos peligros potenciales en caso de una fuga de gas y varian dependiendo de factores como: el tamaño del derrame, las condiciones de almacenamiento, las condiciones ambientales y las características del lugar en el cual se produce el derrame. ${ }^{10}$ Por lo tanto, mientras mayor sea el conocimiento acerca de los peligros asociados a la actividad productiva, de los eventos indeseados que puedan ocurrir, de sus causas y de sus consecuencias, mayor será la capacidad para prevenir su ocurrencia o mitigar sus consecuencias. ${ }^{4}$ Por consiguiente, existen metodologías de evaluación de riesgos que permiten valorar la amenaza que involucra para la salud y seguridad de los trabajadores un determinado peligro en el lugar de trabajo, estimando así el nivel de riesgo, considerando en su conjunto la probabilidad y las consecuencias de que tal peligro se materialice. ${ }^{11}$ La evaluación de riesgos es una herramienta que permite identificar los peligros y dar a conocer a las personas dónde se localizan los riesgos que pudieran causarles daño y cuál es la situación real para poder tomar precauciones. ${ }^{8}$ Existe una amplia gama de métodos utilizados en la industria de procesos; algunos permiten realizar un análisis preliminar de riesgo o una evaluación con mayor nivel de detalle y otros, estimar y jerarquizar los riesgos asociados a un proceso $^{12}$, en donde el método dependerá del objetivo que tenga el análisis. El Programa de las Naciones Unidas para el Medio Ambiente (PNUMA), creó el Programa de Concientización y Preparación para Emergencias a Nivel Local denominado APELL. Éste es un programa dirigido a mejorar la prevención de accidentes tecnológicos y la preparación para su atención. ${ }^{13}$ Por otra parte, el Instituto Nacional de Seguridad e Higiene en el Trabajo (INSHT) de España, publicó la Nota Técnica de Prevención $N^{\circ}$ 937, que trata sobre la utilización de modelos cualitativos o simplificados para la evaluación de la exposición inhalatoria de agentes químicos ${ }^{14}$, y que es útil para realizar un diagnóstico a partir de una serie de variables. $^{15}$

Teniendo en cuenta lo anterior, en este estudio se evalúan los riesgos hacia los trabajadores y la comunidad, asociados a liberaciones accidentales de gases densos peligrosos, desde fuentes de uso y almacenamiento, en la ciudad de Los Ángeles (Chile), ya que existen instalaciones que podrían presentar un potencial peligro de toxicidad hacia las personas, a causa de la inhalación de agentes tóxicos.

\section{MATERIALES Y MÉTODOS}

\section{Área de estudio}

El estudio se lleva a cabo en la zona urbana de la ciudad de Los Ángeles, específicamente, en instalaciones productivas o de servicios que usan y/o almacenan gases densos peligrosos, los que en caso de una liberación accidental pudieran generar toxicidad aguda por medio de la inhalación, tanto a los trabajadores como a la comunidad.

La ciudad de Los Ángeles está ubicada en la octava región, provincia del Biobío, su población comunal es de 187.494 habitantes, encontrándose un 73\% en el área urbana. ${ }^{16}$ El estudio se centró en esta área, debido a la mayor concentración de personas junto con una gran cantidad de instalaciones de almacenamiento y uso de sustancias peligrosas.

\section{INSTRUMENTOS Y PROCEDIMIENTO}

\section{Obtención de información}

Para la identificación de riesgos, primero se realiza una recopilación de información de las actividades productivas presentes en la ciudad y de los gases que éstas usan y/o almacenan en sus instalaciones a través de consultas a organizaciones, portales de negocios y organismos del Estado que cuentan con antecedentes referidos a sustancias químicas y gases combustibles. Algunos fueron: el Ministerio de Salud (MINSAL); la Secretaria Regional Ministerial (SEREMI) de Salud Provincial del Biobío: el Instituto Nacional de Estadísticas, el Ministerio de Energía; la Superintendencia de Electricidad y Combustibles (SEC); y, el Ministerio de Medio Ambiente.

A las actividades productivas identificadas en el paso anterior se les solicita información, con el objeto de complementar los datos necesarios para el estudio. Además, se acude a las Hojas de Datos de Seguridad (HDS) de las sustancias utilizadas y a un Manual de Gases $^{17}$ para conocer sus características, propiedades físicoquímicas, toxicológicas y ecotoxicológicas.

Luego, se lleva a cabo un levantamiento georreferenciado de la zona urbana para obtener información complementaria y se ratifica en terreno la situación de las instalaciones de almacenamiento de gases densos.

\section{Análisis de riesgo}

i) Basado en la Nota Técnica de Prevención $N^{\circ} 937$ de España Para obtener un diagnóstico inicial de las fuentes de uso y/o almacenamiento y del riesgo hacia los trabajadores en caso de inhalación, se utiliza el método basado en el INRS, publicado por el INSHT en la NTP $N^{\circ} 937$ y se evalúa en las siguientes situaciones:

a) Almacenamiento en estanques de Gas Licuado de Petróleo (GLP): se consideraron estanques de $2 \mathrm{~m}^{3}$ o mayores y los puestos de trabajo ubicados en un radio de 100 metros de las instalaciones. De la observación en terreno se determina que estos eran: guardias de seguridad, porteros, jardineros, asesoras de hogar, vendedores de gas, operadores y ayudantes de los operadores a cargo de distribuir GLP a granel, durante su jornada laboral diaria de 8 horas.

b) Gases densos almacenados por las comercializadoras de gases industriales: se consideraron los aspectos de capacidad volumétrica de 50 litros para los cilindros, que los trabajadores expuestos son los encargados de manipular, almacenar y distribuir los cilindros de gas hasta su destino, una frecuencia diaria de utilización de la sustancia para una jornada laboral de 8 horas y la mayor densidad del gas publicada en diferentes Hojas de Datos de Seguridad (HDS).

Para las situaciones a) y b) anteriores, se realiza una evaluación simplificada de riesgos, considerando que los procedimientos de 
trabajo se realizan en un medio dispersivo, simulando una fuga de gas denso y mecanismos de ventilación de acuerdo a la clasificación entregada por la metodología; teniendo en cuenta cuatro variables (riesgo potencial, volatilidad, procedimiento de trabajo $\mathrm{y}$ protecciones colectivas) y un factor de corrección ( $\left.\mathrm{FC}_{\mathrm{VLA}}\right)$ cuando el valor límite ambiental del agente químico fuese muy pequeño, inferior a $0,1 \mathrm{mg} / \mathrm{m}^{3}$. Para cada variable se establecieron clases y una puntuación asociada a cada clase. La puntuación del riesgo se calcula a partir de la puntuación obtenida para cada variable y del factor de corrección aplicado para cada gas almacenado. En la Figura 1 se muestra el esquema aplicado a dicha evaluación.

Figura 1.

Esquema para la evaluación simplificada del riesgo por inhalación.

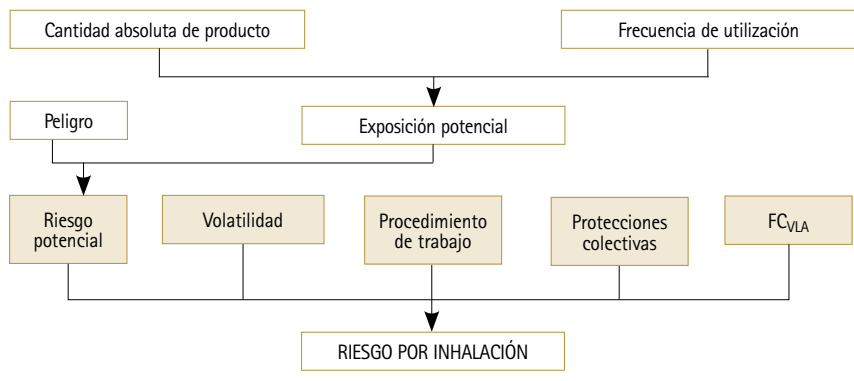

La puntuación del riesgo por inhalación se calcula a través de la siguiente fórmula:

$$
P_{\text {inh }}=P_{\text {riesgo pot. }} \times P_{\text {volatilidad }} \times P_{\text {proced }} \times P_{\text {prot.colec. }} \times F C_{V L A}
$$

Donde:

$\mathrm{P}_{\text {inh }}$ : puntuación del riesgo por inhalación; $\mathrm{P}_{\text {riesgo pot.: }}$ puntaje del riesgo potencial; $\mathrm{P}_{\text {volatilidad }}$ puntaje de las características de volatilidad de la sustancia; $\mathrm{P}_{\text {proced }}$ : puntaje según el procedimiento de utilización; $\mathrm{P}_{\text {prot.colec: }}$ puntaje de la protección colectiva; $\mathrm{FC}_{V L A}$ : factor de corrección del Valor Límite Ambiental. Según la puntuación del riesgo por inhalación obtenida en cada una de las situaciones, se caracteriza el nivel de riesgo en: riesgo a priori bajo, moderado o probablemente muy elevado.

ii) Método basado en el Programa de Concientización y Preparación para Emergencias a Nivel Local (APELL) del PNUMA ${ }^{18}$

Para el análisis de riesgos de aquellas fuentes con potencial daño a las personas (comunidad y trabajadores), se utiliza el método para evaluar e identificar los peligros considerado en APELL, mediante un inventario de identificación y evaluación de riesgos de los objetos riesgosos que almacenan gas denso. Para completar este inventario, se considera la localización de los objetos riesgosos y peligros asociados, los tipos de peligros existentes, los peligros y zonas de riesgo al interior y exterior del sitio en relación con los objetos amenazados (personas), la asignación de un rango a los objetos riesgosos de acuerdo a la probabilidad de ocurrencia de un accidente y la estimación de sus consecuencias para las personas.

Dentro de este marco, para el análisis de los objetos amenazados y/o peligros aislados se utilizan las clasificaciones otorgadas por esta metodología, en cuanto a las consecuencias de un potencial accidente: Poco importantes, Limitadas, Graves, Muy graves y Catastróficas; la velocidad en que se pueda desarrollar; y la probabilidad de que éste se materialice: Improbable, Bastante probable y Muy probable.

Una vez obtenidos los resultados de los análisis de riesgos, éstos se expresan en un mapa a modo de presentar un resumen de los resultados obtenidos, para lo cual se utiliza la aplicación, Google Earth Pro.

\section{RESULTADOS}

\section{Almacenamiento y uso de sustancias peligrosas}

De las diversas fuentes de información y verificación de datos, pudo establecerse que en la ciudad de Los Ángeles existen dos instalaciones que almacenaban gases de uso industrial, dedicados a su comercialización. Una, ubicada en las coordenadas geográficas $37^{\circ} 26^{\prime} 43.36^{\prime \prime}$ Sur y $72^{\circ} 19^{\prime} 37.59^{\prime \prime}$ Oeste a 157 msnm; y otra, con coordenadas $37^{\circ} 24^{\prime} 32.70^{\prime \prime}$ Sur, 72 20'47.30” Oeste, a 145 msnm. De los gases almacenados por estas comercializadoras, se consideraron por su densidad especifica superior a 1, el $\mathrm{Ar}, \mathrm{CO}_{2}$, $\mathrm{N}_{2} \mathrm{O}$ y el $\mathrm{C}_{2} \mathrm{H}_{2}$. También se obtuvo que en la ciudad se almacena GLP de distribución masiva en estanques de diversos tamaños. Se registraron un total de 113 estanques de GLP, cuya distribución por tamaños se muestra en la Figura 2.

\section{Figura 2.}

Estanques de GLP en la ciudad de Los Ángeles según capacidad de almacenamiento.

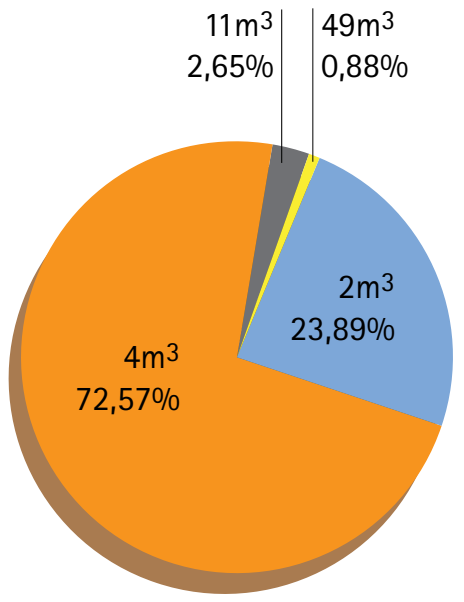

Análisis de riesgo por inhalación en locales de venta de gases industriales basado en la NTP $\mathrm{N}^{\circ} 937$

Se obtuvo que los 4 gases identificados en las comercializadoras de gases presentaron un nivel de riesgo a priori bajo, por lo que no requiere de modificaciones o medidas correctoras, considerándose que, en el caso de una fuga de gas, el mecanismo de trabajo es dispersivo; esto es, que el trabajador se encuentra alejado de la fuente de emisión; o bien, que realiza el trabajo a la intemperie. En la Tabla 1 se muestran las puntuaciones obtenidas del análisis de riesgos para cada gas denso manipulado en ambas comercializadoras.

Tabla 1.

Caracterización del riesgo en comercializadoras de gases industriales.

$\begin{array}{cc}\text { Producto químico } & \text { Puntuación del riesgo } \\ \mathrm{Ar} & 0,7 \\ \mathrm{CO} 2 & 7 \\ \mathrm{~N} 2 \mathrm{O} & 70 \\ \mathrm{C} 2 \mathrm{H} 2 & 7\end{array}$


Análisis de riesgo por inhalación de gas licuado de petróleo (GLP) de distribución masiva basado en la NTP N 937

En la Tabla 2, se presentan los resultados del análisis de riesgo en caso de inhalación de GLP, donde se observa que la caracterización del riesgo para los seis puestos de trabajo analizados resulta ser riesgo moderado, lo que significa que probablemente se necesitan medidas correctoras y/o una evaluación más detallada (mediciones).

Tabla 2.

Puntuación del riesgo según condiciones de trabajo.

$\begin{array}{|ccc|}\mathbf{N}^{\circ} & \text { Puesto de trabajo } & \text { Puntuación del riesgo } \\ 1 & \text { Operador de carga/descarga de gas } & 700 \\ 2 & \text { Ayudante de carga/descarga de gas } & 700 \\ 3 & \text { Guardia de seguridad/portero } & 700 \\ 4 & \text { Vendedor de gas en distribuidora } & 700 \\ 5 & \text { Jardinero } & 700 \\ 6 & \text { Asesora de hogar } & 1000\end{array}$

Análisis de riesgo por fuga de gas en instalaciones de gas licuado de petróleo con la metodología del Programa APELL Se analizaron 79 objetos de riesgo del total del inventario de riesgos correspondientes a instalaciones de GLP, ya que con el resultado de los dos análisis anteriores se descartaron los otros tipos de gases debido a que el nivel de riesgo para ellos resultó ser a priori bajo. Se obtuvo que el 1,27\% de los objetos riesgosos (instalaciones) presentó una probabilidad de ocurrencia clase 4, es decir, una frecuencia estimada de una vez entre 1 a 10 años aproximadamente, mientras que un $65,82 \%$ de las instalaciones analizadas presentó una probabilidad de ocurrencia clase 2, con una frecuencia estimada de una vez entre 100 a 1000 años en caso de ocurrir una fuga accidental de gas (Tabla 3).

Tabla 3.

Objetos riesgosos según probabilidad y frecuencia.

\begin{tabular}{llcc}
$\begin{array}{l}\text { Clase } \\
\text { Probabilidad }\end{array}$ & $\begin{array}{l}\text { Frecuencia estimada } \\
\text { aproximada }\end{array}$ & $\begin{array}{c}\mathrm{N}^{\circ} \\
\text { Objetos }\end{array}$ & $\%$ \\
\hline 5. Muy Probable & Más de una vez por año & 0 & 0,00 \\
4. & Una vez entre 1-10 años & 1 & 1,27 \\
3. Bastante probable & Una vez entre 10-100 años & 26 & 32,91 \\
2. & Una vez entre 100-1000 años & 52 & 65,82 \\
1. Improbable & Menos de una vez por 1000 años & 0 & 0,00 \\
Total & $\mathbf{7 9}$ & 100 &
\end{tabular}

En cuanto a las consecuencias y la prioridad del riesgo por almacenar gas licuado (Tabla 4), se obtuvo que un 3,8\% de los objetos riesgosos y operaciones puede ocasionar consecuencias muy graves para la comunidad y trabajadores, en comparación con un $67,09 \%$ que puede presentar consecuencias limitadas, situación donde los involucrados pueden presentar incomodidad permanente por liberación del gas.

Tabla 4.

Clasificación de riesgos según prioridad y consecuencias.

\begin{tabular}{llcc} 
Prioridad & Consecuencias & Nºbjetos $^{\circ}$ & $\%$ \\
E & Catastróficas & 0 & 0,00 \\
D & Muy graves & 3 & 3,80 \\
C & Graves & 23 & 29,11 \\
B & Limitadas & 53 & 67,09 \\
A & Poco importantes & 0 & 0,00 \\
Total & $\mathbf{7 9}$ & 100 & \\
\hline
\end{tabular}

De acuerdo a los resultados obtenidos en los análisis, junto con las coordenadas geográficas registradas durante la constatación en terreno, se logró posicionar la ubicación de cada una de estas instalaciones e indicar la prioridad del riesgo estimado en caso de una liberación accidental de gases.

En el mapa, según la clasificación de riesgo de APELL, se obtuvieron tres niveles: $\mathrm{B}, \mathrm{C}$ y D, considerando las posibles consecuencias que una fuga de gas podria provocar en los trabajadores y habitantes de la ciudad. Éste se clasificó según los barrios urbanos, obteniéndose que la mayor cantidad de objetos riesgosos se encuentran en el barrio Alemania, seguido por el barrio Sor Vicenta (Figura 3).

Figura 3.

Objetos riesgosos según barrio de zona urbana de la ciudad.

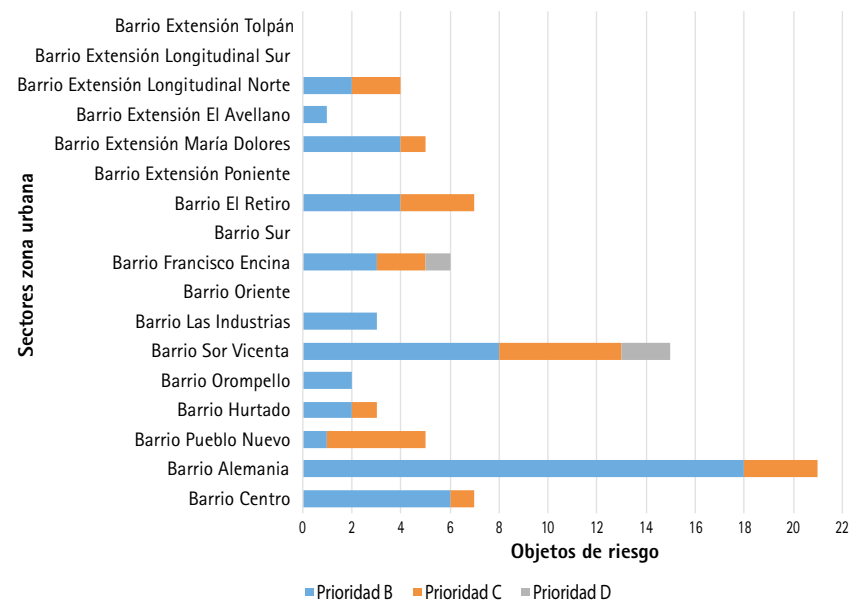

En la Figura 4, se observa que los 3 objetos riesgosos con prioridad clase D poseen consecuencias estimadas muy graves, debido que allí se ubican estanques de gran capacidad, 11 y $49 \mathrm{~m}^{3}$, pertenecientes a las distribuidoras de gas ubicadas en los sectores urbanos Sor Vicenta y Francisco Encina respectivamente, las cuales, debido al crecimiento de la población, se encuentran cada vez más rodeadas de conjuntos habitacionales.

En la Figura 5, se observa las instalaciones que poseen una clasificación de riesgos clase $\mathrm{C}$, con consecuencias estimadas graves tanto para los trabajadores y comunidad. Este tipo de instalaciones se caracterizó por encontrarse rodeadas por una gran cantidad de personas, pudiendo ser centros educacionales, tienda de materiales de construcción, supermercados, entre otras.

Figura 4.

Mapa de riesgos con instalaciones de GLP con prioridad de riesgo clase D.

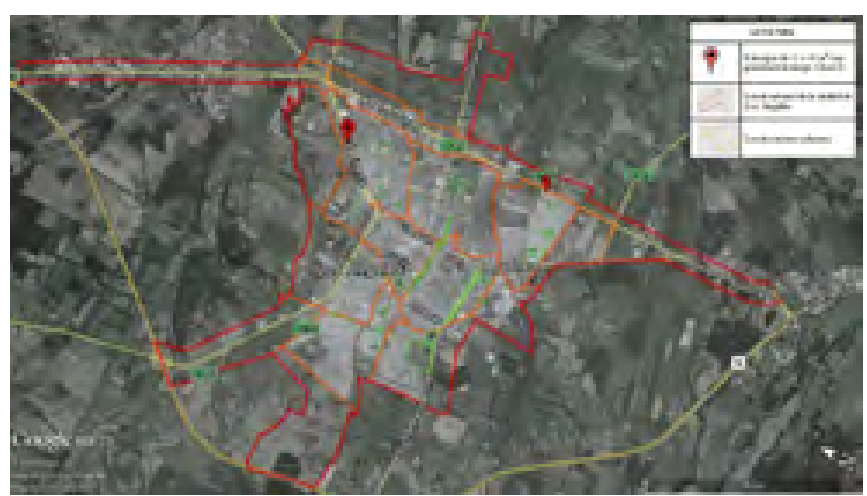


Figura 5.

Mapa de riesgos con instalaciones de GLP con prioridad de riesgo clase C.

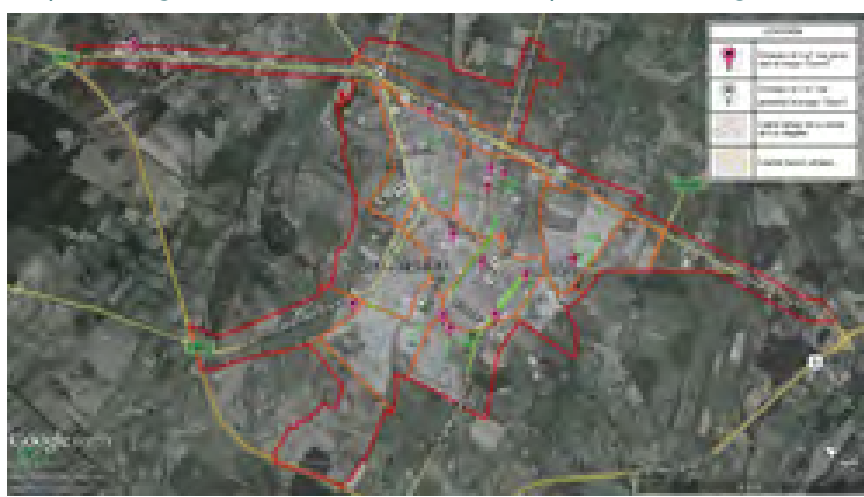

Finalmente, en la Figura 6, se observa una mayor cantidad de instalaciones con prioridad de riesgo clase $\mathrm{B}$, es decir, que estos objetos pueden provocar consecuencias limitadas para las personas, ya que según lo observado en terreno, muchos de éstos se encuentran con los sellos de seguridad en condiciones óptimas y sin daños.

Figura 6.

Mapa de riesgos con instalaciones de GLP con prioridad de riesgos clase $B$.

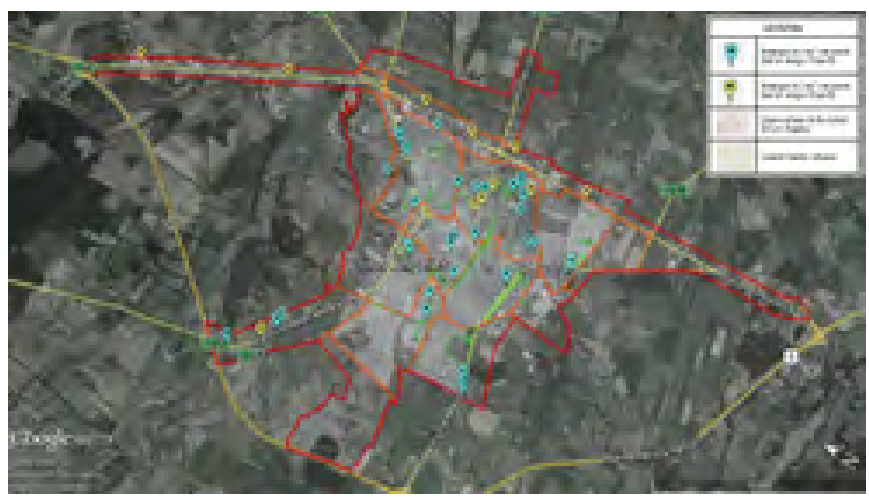

\section{DISCUSIÓN}

Un estudio realizado por Sarmiento ${ }^{19}$ sobre emergencias ambientales asociadas a productos químicos manifiesta que: el $80 \%$ de éstas corresponden a fugas (gases) y derrames (líquidos), seguidos de los incendios, las explosiones y otro tipo de eventos y que la principal preocupación de las fugas radica en el riesgo que puede afectar la integridad y la salud de las personas, lo cual se relaciona con la importancia de los resultados obtenidos en este análisis, ya que conocer el nivel de riesgo existente de cada fuente permite prevenir o controlar la ocurrencia de posibles emergencias tanto al interior como al exterior de un recinto.

Por otra parte, el uso y almacenamiento de estanques de GLP con capacidad de $4 \mathrm{~m}^{3}$, se asoció a la gran cantidad de conjuntos habitacionales presentes en la zona urbana de la ciudad, junto con su utilización por empresas del rubro industrial y comercial, donde la capacidad almacenada se correlaciona con el nivel de consumo diario/mensual de las instalaciones. Esto influye en el uso de estanques móviles de mayor capacidad, encargados de abastecer el gas a granel por las distintas instalaciones. Dentro de este marco,
Villafañe ${ }^{10}$ expone que el sector domiciliario alcanza el 45\% de la demanda mundial de GLP, y que uno de los sectores con mayor crecimiento ha sido el transporte, representando casi el 9\% del consumo mundial, durante el año 2011. En tanto que en Chile en los informes anuales de la SEC indican que el consumo en la región del Biobío aumentó un 28\% entre los años 2006 y $2014^{20}$, no encontrándose datos estadísticos sobre el comportamiento del consumo de gas a nivel de la ciudad de Los Ángeles.

Finalmente, la representación de los riesgos en un mapa permite conocer los objetos vulnerables existentes alrededor de un objeto riesgoso y aquellos elementos que pueden verse afectados ante un accidente, así como también conocer las vías de transporte existentes $^{21}$, ya que es de vital importancia que en caso de una emergencia las personas involucradas conozcan de antemano las rutas óptimas para la llegada de equipos de rescate y puedan evacuar oportunamente.

\section{CONCLUSIONES}

En esta investigación se concluye lo siguiente:

- En la zona de estudio, se encuentran comercializadoras de gases industriales y distribuidoras de GLP que almacenan gases más densos que el aire.

- El riesgo de inhalación accidental de gases densos en las comercializadoras de gases industriales resulta ser siempre a priori bajo, es decir, no se necesita realizar modificaciones a las variables estudiadas, mientras que en las distribuidoras e instalaciones de GLP el riesgo es siempre moderado para los trabajadores, es decir, que probablemente se necesiten medidas correctoras y/o una evaluación más detallada.

- Según el análisis con la metodología de evaluación de riesgos de APELL, la mayoría de los estanques de almacenamiento de GLP no presenta un riesgo alto para la comunidad. Las consecuencias más graves en caso de una fuga pueden ocurrir en las distribuidoras de gas licuado, ya que en éstas se almacena gas en estanques con una gran capacidad y porque se encuentran cada vez rodeadas de más personas debido al crecimiento de la población.

- El almacenamiento y transporte de gases densos peligrosos está en aumento, es por ello que conocer el nivel de riesgo asociado a cada instalación en un mapa georrefenciado permite conocer las rutas de acceso en caso de emergencias, la ubicación de las instalaciones y los objetos de riesgo amenazados.

- Los objetos de riesgos evaluados se concentran mayormente en barrios destinados para residencia o conjuntos habitacionales; sin embargo, en los barrios Sor Vicenta y Francisco Encina existen situaciones donde la clasificación del riesgo es muy grave, debido a la cantidad de gas almacenado y la exposición de los trabajadores y habitantes durante el traslado de gas licuado.

- La información que entrega este estudio sirve de base para la toma de decisiones políticas o de inversión en Planes de Prevención o Control de Emergencias en la cuidad que pueden ser utilizadas por diversos Organismos Públicos, Organismos No Gubernamentales, industrias y comunidad en general.

\section{Agradecimientos}

Los autores agradecen particularmente a la Vicerrectoría de Investigación y Desarrollo por los recursos entregados a través del proyecto VRID N²15.418.008-1.0 IN. 


\section{REFERENCES}

1. Programa de las Naciones Unidas para el Medio Ambiente-PNUMA. Marco Flexible para la Prevención y Preparación en Accidentes en caso de Accidentes con Productos Químicos. Paris: UNEP; 2010.

2. Lavell A. Sobre la Gestión del Riesgo: Apuntes hacia una Definición [en línea]. (s.n.); 2001. [consultado abr 2015]. Disponible en: http://microseguros.net/ seminario/biblioteca/vulnerabilidad/doc15036-lavell-gestion-riesgo.pdf

3. Amparo G. Conflictos ambientales amenazan la salud de la población y la biodiversidad del Planeta. Rev Derecho (Barranquilla). 2007;(28):329-347.

4. Márquez F. Principios de Análisis de Riesgo para la Industria de Procesos. Concepción: Universidad de Concepción; 2009.

5. Baxter P. Enciclopedia de Salud y Seguridad del Trabajo. Vol. II. Preparación para las catástrofes. Madrid: OIT-INSHT; 1999. p.15-20.

6. Turmo E, Cuscó J. NTP N 329. Modelos de dispersión de gases y/o vapores en la atmósfera: fuentes puntuales continuas. Madrid: Instituto Nacional de Seguridad e Higiene en el Trabajo; 1999?

7. Carrari C, Aparicio L, Bandoni J, Tonelli S. Utilización de modelos de dispersión atmosférica para la estimación de dosis de exposición. Mecánica Computacional. 2004; 23:1743-1759.

8. Díaz C, Vázquez R, Mannan M. Determinación de los factores del peor escenario en la emisión de gases tóxicos. Inf Tecnol. 2009;20(1):3-10.

9. Menéndez C. Notas para el Análisis de Riesgos en la Industria de Procesos. Cuba: Instituto Superior Politécnico José Antonio Echeverria; 2007.

10. Villafañe D. Estudio de la dispersión e incendio de nubes inflamables de gas (GNL y GLP) [Tesis doctoral]. Barcelona: Universitat Politècnica de Catalunya; 2013.

11. Cortés J. Evaluación de riesgos. En: Seguridad e Higiene del Trabajo. Madrid: Editorial Tébar S.L. 2007;9:123-134.

12. Zaror C. Introducción a la Ingeniería Ambiental para la Industria de Procesos. Chile: Universidad de Concepción; 2000.

13. Programa de las Naciones Unidas para el Medio Ambiente (PNUMA). TransA-
PELL: una guia de planeación para emergencias durante el transporte de materiales peligrosos en una comunidad local. Francia: PNUMA/ORPALC; 2000. Informe Técnico $N^{\circ} 35$.

14. Cavallé N. NTP 935 Agentes Químicos: Evaluación cualitativa y simplificada del riesgo por inhalación (I): Aspectos Generales. Madrid: Instituto Nacional de Seguridad e Higiene en el Trabajo; 2012.

15. Sousa M, Tejedor J. NTP $N^{\circ}$ 937. Agentes químicos: evaluación cualitativa y simplificada del riesgo por inhalación (III): Método basado en el INRS. Madrid: Instituto Nacional de Seguridad e Higiene en el Trabajo; 2012.

16. Instituto Nacional de Estadisticas (INE). Resultados XVIII Censo de Población: Caracteristicas demográficas, sociales, culturales y económicas de la población [Internet]. Tomo I. Santiago: INE; 2012. [accesado 14 de diciembre 2015] Disponible en: http://www.cooperativa.cl/noticias/site/artic/ 20130425/asocfile/20130425190105/resultados_censo_2012_poblacion_ vivienda_tomosiyii.pdf

17. Indura. Manual de Gases [Internet]. Chile: Indura; 2015. [accesado 14 de diciembre 2015]. Disponible en: http://www.indura.net/content/storage/ec/bibl ioteca/115c34ca0e684d41b098c9fbbc861cac.pdf

18. Programa de las Naciones Unidas para el Medio Ambiente (PNUMA). Identificación y Evaluación de Riesgos en una Comunidad Local (2da ed.). México: Universidad de Guadalajara; 2003.

19. Sarmiento M. Emergencias ambientales asociadas a sustancias químicas en México. Gaceta Ecológica. Ene-mar 2003;(66):54-66. ISSN: 1405-2849.

20. Superintendencia de Electricidad y Combustibles (SEC). Informe estadístico. [Internet]. Chile: SEC; 2015. [accesado 22 de diciembre 2015]. Disponible en http://www.sec.cl/portal/page?_pageid=33,3429539\&_dad=portal\&_ schema=PORTAL

21. Delgado R, González P. El riesgo de desastre químico como cuestión de salud pública. Rev Esp Salud Pública. Nov-Dic 1998; 72 (6): 481-500. 